\title{
e-Migrinter
}

$22 \mid 2021$

Varia

\section{Les jeunes migrant·es et l'École : parcours et enjeux de scolarisation}

Compte-rendu de la table ronde conclusive du cycle de conférences « Migrations et cycle de vie » organisé par l'Espace Mendès France, Migrinter, et des étudiantes du Master Migrations Internationales, le 25 mars 2021

Lola Bultel, Bonnie Couillet, Lise Fauvre et Luna Russo

\section{(2) OpenEdition}

Journals

Édition électronique

URL : https://journals.openedition.org/e-migrinter/2765

DOI : 10.4000/e-migrinter.2765

ISSN : 1961-9685

Éditeur

UMR 7301 - Migrinter

Référence électronique

Lola Bultel, Bonnie Couillet, Lise Fauvre et Luna Russo, « Les jeunes migrant·es et l'École : parcours et enjeux de scolarisation », e-Migrinter [En ligne], 22 | 2021, mis en ligne le 15 décembre 2021, consulté le 17 décembre 2021. URL : http://journals.openedition.org/e-migrinter/2765 ; DOI : https://doi.org/ 10.4000/e-migrinter.2765

Ce document a été généré automatiquement le 17 décembre 2021.

Tous droits réservés 


\section{Les jeunes migrant·es et l'École : parcours et enjeux de scolarisation}

Compte-rendu de la table ronde conclusive du cycle de conférences «Migrations et cycle de vie » organisé par l'Espace Mendès France, Migrinter, et des étudiantes du Master Migrations Internationales, le 25 mars 2021

Lola Bultel, Bonnie Couillet, Lise Fauvre et Luna Russo

\section{RÉFÉRENCE}

«Les jeunes migrant·es et l'École : parcours et enjeux de scolarisation », 25 mars 2021, dans le cadre du cycle de conférence « Migrations et cycle de vie » co-organisé par l'Espace Mendes France et Migrinter

Compte rendu de la table ronde ${ }^{1}$ autour de Virginie Baby-Collin, Professeure de géographie, AixMarseille Université, laboratoire Telemme (CNRS) et Julie Vinot, Professeure de lettres, français langue étrangère et seconde au lycée Pilote innovant international à Jaunay-Marigny, Poitiers, le 25 mars 2021, organisée et animée par les autrices de ce compte-rendu.

Cette conférence a été organisée dans le cadre d'un travail collectif.

Nous souhaitons remercier Virginie, Cécile, Julie pour leur temps, leur bienveillance et leurs précieux conseils.

Nous remercions, pour le partage de leur temps et de leurs paroles, les élèves du LP2i, à savoir, Fatima Naasam, Pengyu Liang, Junior Boris Deffo Talla, Vu Duy Nguyen, Mohamed Touihar, Pouyan Khosravi.

Enfin, un grand merci à Brenda Le Bigot, Jordan Pinel et Héloïse Morel de nous avoir donné cette opportunité et pour leur encadrement. 


\section{Introduction}

1 Cette table ronde a été organisée dans le cadre du cycle de conférences Migrations internationales et lutte contre les discriminations proposé par l'Espace Mendès France et le laboratoire Migrinter de l'Université de Poitiers. Elle découle de la proposition de Brenda Le Bigot et Jordan Pinel, géographes au laboratoire et Héloïse Morel, médiatrice scientifique. C'est en suivant leurs conseils que quatre étudiantes (Lola Bultel, Bonnie Couillet, Lise Fauvre, Luna Russo) du master Migrations Internationales et membres de l'Association Migr'Avenir se sont saisies du projet. La thématique centrale du cycle de conférence depuis la rentrée 2019 étant " Migrer tout au long de la vie », nous avions envie de partager une thématique importante : la scolarisation des élèves étrangeres en France.

2 Cette table ronde visait à mettre en lumière des terrains et approches différents, à travers le point de vue des sciences sociales, des professionnelles de terrain et des jeunes concernées. Pour cela, une chercheuse et une enseignante coordinatrice d'une Unité pédagogique pour élèves allophones arrivantes (UPE2A) sont intervenues pour mettre en perspective l'hétérogénéité des parcours et des modalités de scolarisation des élèves allophones en France. La première intervenante était Julie Vinot, professeure de lettres, français langue étrangère et seconde au sein du lycée Pilote innovant international à Jaunay-Marigny (près de Poitiers). Elle était accompagnée de Virginie Baby-Collin, Professeure de Géographie à l'Université Aix-Marseille et membre du laboratoire Telemme (CNRS), qui, parmi ses projets de recherche, mène un suivi de scolarisation d'élèves allophones nouvellement arrivées (EANA) scolarisées dans une UPE2A dans un lycée de Marseille. Elle mène cette recherche avec Cécile Exbrayat, enseignante coordinatrice de ce dispositif depuis son ouverture, Luna Russo (doctorante, Telemme et Adef, AMU), Polina Palash (post doctorante, Telemme, AMU). Cette recherche se fonde principalement sur des méthodes qualitatives (entretiens avec élèves et acteur-ices socio-éducatifives, échanges réguliers avec les élèves notamment). Il est important de préciser que le contexte marseillais faisant l'objet d'un suivi particulier, contrairement à celui de Poitiers, nous a permis d'avoir une connaissance du terrain d'un point de vue des sciences sociales.

\section{La scolarisation des élèves allophones nouvellement arrivé·es en France}

3 La scolarisation des élèves migrantes suscite un intérêt croissant depuis une dizaine d'années en sciences humaines et sociales. Ces recherches, à la croisée des enjeux migratoires et des enjeux éducatifs, se multiplient dans un contexte global de développement des travaux sur les migrations mais aussi, parce que le nombre d'élèves migrant·es scolarisées dans les écoles françaises a connu une augmentation au XXI ${ }^{\text {ème }}$ siècle.

4 La présence d'élèves étrangeres à l'école en France n'est pas récente. Leur présence s'est accompagnée de la création de classes ou dispositifs spécifiques - fermés - à partir des années 1970. Dans les années 1980, le nombre d'enfants étrangeres scolarisées a stagné voire diminué. Depuis, ce nombre croît : de 32000 en 2001-2002, ils sont près de 70000 en 2018-2019. Depuis la circulaire n²012-141 (BOEN, 2012), les élèves étranger·es 
sont désignées par le terme d' "élèves allophones nouvellement arrivées » (EANA). Ils et elles se caractérisent par deux éléments principaux : une langue maternelle autre que le français et une arrivée récente sur le territoire français (théoriquement moins d'un an). Ces dernieres sont scolarisées dans des dispositifs - ouverts - appelés les Unités pédagogiques pour élèves allophones arrivant·es (UPE2A, depuis 2012), existant aux niveaux primaire et secondaire. Une minorité d'entre elles et eux est scolarisée en lycée général au sein des UPE2A - c'est ce public que nous souhaitions mettre en avant dans cette table ronde. À travers un apprentissage spécifique du français comme langue de scolarisation et l'inclusion ${ }^{2}$ dans des cours de classes dites ordinaires (durant une année théoriquement) l'UPE2A en lycée général permet aux EANA - de plus de seize ans justifiant d'une scolarité antérieure en collège ou au lycée - de poursuivre leurs études dans le système scolaire français. Cependant, cette scolarisation s'inscrit dans un contexte différent du ou des anciens lieux de vie. Les défis à relever sont nombreux pour ces élèves issu'es de parcours migratoires souvent complexes et heurtés : nouvelle langue de scolarisation mais aussi nouvel établissement, nouveaux modes d'apprentissages, nouveaux codes (Armagnague et al., 2018 ; Guedat-Bittighoffe, 2015).

Pour être scolarisée en France, l'élève allophone de plus de 16 ans doit passer un test de positionnement réalisé par le Centre académique pour la scolarisation des enfants allophones nouvellement arrivées et des enfants issues de familles itinérantes et de voyageurs (CASNAV) et les Centres d'information et d'orientation (CIO). Ce test évalue leur niveau en langue française et leur niveau scolaire. En fonction des résultats, l'élève est affectée dans sa classe d'âge. Certain'es sont affecté'es en dispositif spécifique comme l'UPE2A, d'autres en classe ordinaire sans soutien linguistique spécifique. Une fois que le test est effectué, l'affectation n'est pas immédiate : les délais de prise en charge peuvent être longs (surtout au lycée) et le nombre de places est limité par rapport au nombre d'EANA présentes sur le territoire.

6 Ainsi, la scolarisation des EANA pose de nombreuses questions : la formation des enseignant-es, les pratiques pédagogiques, l'orientation, l'expérience de l'Ecole par les élèves; autant d'enjeux qui ont été abordés par Julie Vinot et Virginie Baby-Collin. Même si des travaux ont été réalisés, peu de données existent à propos de leur orientation, leurs trajectoires et leur réussite scolaire (Caille \& Vallet, 1996 ; Schiff, 2002 ; Felouzis \& Fouquet-Chauprade, 2016 ; Armagnague et al., 2018a, b, 2019).

\section{Mise en lumière de deux dispositifs accueillant des EANA à Poitiers et Marseille}

\section{Contextualisation des territoires et des publics}

7 L'échange entre les deux intervenantes a permis de souligner que, malgré la présence de dynamiques migratoires dans les deux territoires de Marseille et Poitiers, ces derniers portent des réalités migratoires et de scolarisation distinctes.

Virginie Baby-Collin nous a présenté le contexte à Marseille. Depuis une vingtaine d'années, le nombre d'EANA ayant fortement augmenté dans l'académie, de nombreux dispositifs ont été créés. Les dispositifs se concentrent surtout à Marseille $-20^{\text {ème }}$ des 100 plus grandes communes les plus pauvres de France (Audren et al., 2018) - où 80\% des EANA sont scolarisées, et sont surtout dans les réseaux d'éducation prioritaire (REP et REP+). Dans cette académie, la grande majorité des UPE2A sont des UPE2A-Lycée 
professionnel ${ }^{3}$. Celle sur laquelle porte la recherche présentée par Virginie Baby-Collin est l'unique, dans l'académie, à être une UPE2A-Lycée général et technologique (UPE2ALGT) pour laquelle les élèves doivent justifier d'un niveau de scolarisation antérieure équivalent à au moins une fin de Troisième.

9 Cette UPE2A, implantée au lycée Saint-Charles, un établissement central du $1^{\text {er }}$ arrondissement, quartier mixte et espace d'accueil (Ibid), a été créée en 2014 dans un lycée à dimension internationale avec des langues rares comme le russe et l'arménien ainsi que des sections internationales et des classes bilingues. Depuis 2014, c'est la même enseignante, Cécile Exbrayat, qui coordonne le dispositif. Environ 130 élèves, davantage de garçons que de filles, sont passées par cette UPE2A. Ils et elles sont issu'es d'une grande variété de pays (plus de trente) même si deux dominent : l'Algérie et l'Arménie.

10 Notre deuxième intervenante, Julie Vinot, nous a présenté le LP2i, Lycée Pilote Innovant et International, de Jaunay-Marigny, l'établissement dans lequel elle est enseignante coordinatrice de l'UPE2A. Le LP2i est un lycée général et technologique ${ }^{4}$ qui accueille un des deux seuls dispositifs UPE2 $\mathrm{A}^{5}$ niveau lycée de l'académie de Poitiers. En effet, la Vienne se place dans les six départements où les élèves allophones sont les moins scolarisées (moins de 20\%) dans des dispositifs UPE2A (Ministère de l'Éducation nationale de la Jeunesse et des Sport, 2020). Le LP2i est un lycée qui fonctionne depuis sa création dans les années 1980 autour d'une pédagogie dite innovante, axée sur la pédagogie de projets, l'innovation numérique, le décloisonnement, l'accompagnement de l'élève et l'ouverture sur l'étranger. Élèves et adultes tentent de repenser régulièrement les contours de ces dispositifs, mouvants, évoluant au fil des années. L'inclusion des élèves allophones depuis 2007 est l'un des axes des réflexions menées. Bien que le dispositif du lycée LP2i n'ait pas la labellisation de l'UPE2A, le dispositif fonctionne comme tel.

11 Dans les deux établissements, les EANA suivent entre 10 et 16 heures hebdomadaires de français langue seconde et de scolarisation ${ }^{6}$ (FLS), des heures de co-enseignements FLS (histoire-géographie, sciences, philosophie, physique-chimie par exemple) puis des heures en classe ordinaire de Seconde dans laquelle ils et elles sont rattachées (mathématiques, anglais et EPS à Saint-Charles; EPS, LV1, mathématiques, SVT, physique-chimie, histoire-géographie à Jaunay-Marigny) ${ }^{7}$. Ainsi, les EANA passent un grand nombre d'heures "entre eux" et d'autres avec les élèves francophones natifves.

$12 \mathrm{Au}$ LP2i, trois enseignantes (titulaires, professeures de lettres ayant une formation FLS et une certification complémentaire) accompagnent ces élèves l'année de Seconde dans le dispositif puis le long de leur scolarité dans ce lycée, soit leur année de Première et Terminale. La stabilité de ce dispositif n'est pas antinomique avec son caractère mouvant : d'une année à l'autre l'UPE2A change en fonction des nouveaux élèves, des discussions de l'équipe pédagogique mais aussi des heures dont le dispositif sera doté. À Marseille, les élèves passent théoriquement une année dans le dispositif (environ 20\% y reste une seconde année). Et, il y a une seule enseignante référente. Par conséquent, l'accompagnement à la sortie du dispositif se fait de manière plus individualisée et informelle par Cécile Exbrayat. Un des enjeux de Cécile Exbrayat est le suivi de la coordination du dispositif pour que, l'année suivante, soit pris en compte notamment le progrès des élèves en français dans leurs évaluations et orientation scolaire. Leur non maitrise de la langue française ne doit pas être comprise comme un échec scolaire. Ainsi, l'enseignante coordinatrice doit être en constante communication avec les autres 
enseignant-es et acteur.ices éducatifs du lycée (voire d'autres établissements) pour s'assurer la bonne prise en compte de leurs parcours en tant qu'élèves allophones, même après leur sortie du dispositif.

\section{Les enjeux à l'École}

13 A l'occasion de cette conférence, certain'es élèves de l'UPE2A du lycée LP2i ont accepté de partager leurs expériences. Compte tenu de la situation sanitaire, il nous a été impossible de les faire participer à la conférence. Nous avons donc eu l'idée d'un podcast $^{8}$, ensuite diffusé lors de la conférence.

Tout d'abord, les jeunes identifient la langue comme la première barrière pour non seulement suivre au mieux les cours mais aussi dans leur socialisation avec les élèves francophones natif-ves. Pourtant, le cœur du dispositif est d'empêcher ce phénomène. Les enseignantes coordinatrices considèrent qu'une langue n'est pas "hors-sol". Au contraire, elle implique une vision du monde, une histoire collective et personnelle, une charge émotionnelle pour l'élève. Le rôle des enseignantes est donc de former un pont entre le français comme langue parlée à l'école et leur(s) langue(s) maternelle(s). Les élèves allophones ont un répertoire linguistique riche et diversifié (Chnane Davin, 2020) : ils et elles maîtrisent en général au moins deux langues. Dans cette optique, les deux enseignantes mettent en avant deux techniques dans leurs approches pédagogiques. D'un côté, elles font en sorte que les enseignements en UPE2A valorisent les langues maternelles des élèves et les histoires qu'elles portent. Par exemple, la lecture et traduction d'un texte d'une langue maternelle au français peut laisser l'espace à un débat plurilingue sur l'interprétation de ce texte. Le français est donc enseigné dans toutes ses dimensions et en lien avec d'autres langues. Ces éléments assurent que le français ne soit pas imposé comme un outil « d'acculturation ». D'un autre côté, les enseignantes mobilisent de nombreux types de matériaux et/ou d'activités pour motiver à l'apprentissage de la langue. Au LP2i, se mettent en place des activités avec les élèves de classes dites ordinaires grâce notamment à des collectifs artistiques comme $\mathrm{ACTE}^{9}$; à Saint-Charles, Cécile Exbrayat travaille avec l'Association pour la promotion de la traduction littéraire ${ }^{10}$, avec laquelle les élèves réalisent un travail de traduction d'un texte en français dans leur(s) langue(s) maternelle(s) ou inversement. Le but étant que les élèves débattent de leur interprétation sur la traduction.

Un deuxième enjeu relevé est l'isolement du dispositif dans les établissements. L'École étant l'un des pôles traditionnels de socialisation pour toutes les jeunes, elle l'est d'autant plus pour les élèves allophones qui, suite à une récente arrivée, ont peu de repères (Baby-Collin et al., à paraître en 2021). Il est donc central de penser l'inclusion de ces dernieres au sein de cette institution. Les élèves du LP2i nous ont fait part de leurs difficultés concrètes à tisser des liens au sein de l'établissement scolaire, et ce, malgré le suivi de cours avec des élèves francophones natifves. Cet isolement touche le dispositif de manière générale. Dans les textes (BOEN, 2012), la scolarisation des élèves allophones doit concerner tou-tes les acteurices socio-éducatifs des établissements. Mais, les enseignant·es des dispositifs se retrouvent, très souvent, à devoir gérer seules les élèves (Rigoni, 2020). Ce sont elles et eux qui prennent en charge la construction d'une politique d'inclusion au sein des établissements. Au LP2i, le personnel de l'établissement se retrouve autour de l'objectif commun de construire une ouverture à 
l'international qui permet de ne pas isoler le travail des enseigantes de l'UPE2A et de trouver du soutien et de la concertation collective. Au lycée Saint-Charles, Cécile Exbrayat n'a pas autant de soutien dans l'établissement et se bat avec d'autres enseignantes pour atténuer l'isolement. Finalement, l'effectivité de l'inclusion scolaire repose fortement sur des budgets, matériels et la volonté des personnels, aléatoires selon les établissements.

Un troisième enjeu soulevé est l'engagement, le dépassement des fonctions purement scolaires des enseignantes face à ce public. Étant les premières interlocutrices des élèves allophones et de leurs familles, les enseignantes coordinateur.ices ont une considération globale des jeunes et de leurs conditions de vie en dehors de l'école. Sans que cela soit la seule cause de précarisation, les parcours migratoires et administratifs de ces jeunes impactent les conditions de vie et par conséquent leur parcours scolaire (Armagnague et al., 2018).

17 Leurs parcours migratoires sont hétérogènes, ils et elles viennent de différents pays, de différents milieux socio-économiques ; certaines ont séjourné dans des camps humanitaires, d'autres sont arrivées en famille ou seul-es ; certain'es étaient scolarisées avant de migrer, d'autres non. Julie Vinot a fait part de ces difficultés. Par exemple, les enfants et leur famille sont fréquemment préoccupées par la régularisation de leur statut juridique et la menace d'une Obligation de Quitter le Territoire (OQTF). Comme Cécile Exbrayat, elles font face à des questions très techniques telles que le besoin de faire une demande d'intervention d'une interprète pour communiquer avec la famille, la prise en compte de la situation administrative de l'élève, qui implique des rendezvous à la Préfecture ou encore à l'office français de l'immigration et de l'intégration (OFII). De plus, leur situation résidentielle peut parfois aussi être instable. Certaines sont hébergées en hôtel social ou se retrouvent sans domicile fixe à défaut d'être pris en charge dans des structures spécifiques (Bouillon et al., 2019). Ces situations peuvent perturber leur bien être psychologique, leur faire manquer des cours, être en retard ; et posent des questions en termes de formation des enseignantes à ces problématiques et d'engagement émotionnel.

Ce constat conduit à s'interroger sur les marges de manœuvre et les responsabilités informelles ou "hors contrat" du corps enseignant, qui fait face à des situations qui dépassent leur rôle strictement éducatif (Mendonça Dias \& Rigoni, 2019). L'engagement associatif en parallèle du statut d'enseignante est une des options choisies pour y faire face. Julie Vinot précise que le travail associatif va se placer du côté de la vie personnelle. Face aux manquements de l'État, il est nécessaire de se tourner vers les associations et acteurs non-institutionnels. Le lycée Saint-Charles est notamment en lien avec Réseau Éducation Sans Frontières (RESF). Ensemble, ils organisent des collectes au sein du lycée et permettent par exemple le financement de nuits à l'hôtel pour certaines jeunes. Il s'agit là d'un investissement fort de la part des enseignantes, en termes de temps mais aussi d'affect (Azaoui, 2020).

Dans un dernier temps, les intervenantes ont fait part des enjeux liés à l'orientation scolaire. Elles ont rappelé que l'UPE2A a vocation à ce que les élèves suivent une scolarité ordinaire, et doivent donc s'orienter après l'année de Seconde. Plusieurs choix de poursuite de scolarité sont possibles : intégrer une filière générale, technologique ou professionnelle, en faisant une Seconde ordinaire ou directement une Première. Le projet d'orientation se construit dès le début de l'année et se peaufine tout le long de 
l'année. Les enseignantes refusent une orientation par défaut et font un travail d'orientation dans lequel l'élève est acteur.

Julie Vinot et Virginie Baby-Collin ont questionné les effets de la spécificité d'être nouvellement arrivées sur la construction de leur orientation scolaire. Plusieurs éléments entrent en compte tels que les politiques migratoires et les critères de régularisation, la situation pré-migratoire, le rôle de l'École dans la reproduction des discriminations systémiques, les conditions d'installation. Un des éléments particuliers à prendre en compte est leur maitrise des langues. On l'a vu, les enseignantes coordinatrices mènent une politique de valorisation du plurilinguisme des élèves allophones qui doit être un atout dans l'orientation. Cependant, ce n'est pas si simple : il est difficile de faire en sorte que leur langue maternelle soit une deuxième langue possible à présenter aux épreuves du Baccalauréat. Donc, certaines jeunes doivent apprendre l'espagnol, par exemple - en plus du français et de l'anglais qu'ils et elles apprennent déjà - alors qu'ils et elles en maîtrisent d'autres. Dans ce contexte, les enseignantes luttent pour laisser la possibilité aux élèves allophones de suivre des cours au Centre national d'enseignement à distance (CNED) ${ }^{11}$. Loin d'être simple, l'inscription au CNED dépend non seulement de la volonté et des moyens de la direction des lycées mais aussi du choix de langues au CNED.

\section{Conclusion}

21 La scolarisation des enfants allophones nouvellement arrivées fait l'objet de nombreux enjeux. Alors que l'École est un lieu principal de socialisation, les dispositifs mis en place pour accueillir ces élèves posent notamment la question de l'inclusion scolaire. Recueillir la parole de ces jeunes a permis de rendre compte des obstacles auxquels ils et elles doivent parfois faire face au sein de leur établissement. La langue est une des premières barrières, elle s'accompagne de l'isolement et de la difficulté de l'orientation. Les témoignages de Virginie Baby-Collin et Julie Vinot prouvent que l'engagement des enseignant-es joue un rôle essentiel dans le parcours scolaire de ces élèves, tout comme la volonté de l'établissement de leur faire une place.

Cette conférence a permis, plus généralement, de souligner l'importance de réseaux entre les différents "soutiens" (scolaire, associatif, juridique, d'hébergement, familles) des jeunes migrantes mais aussi avec les mondes de la recherche en sciences sociales.

\section{BIBLIOGRAPHIE}

Armagnague-Roucher, Maitena ; Rigoni, Isabelle ; Cossée, Claire ; Mendonça Dias, Catherine ; Tersigni, Simona (2018) Rapport de recherche EVASCOL. Étude sur la scolarisation des élèves allophones nouvellement arrivés (EANA) et des enfants issus de familles itinérantes et de voyageurs (EFIV). Défenseur des Droits. INSHEA - Institut national supérieur de formation et de recherche pour l'éducation des jeunes handicapés et les enseignements adaptés. https://halshs.archivesouvertes.fr/halshs-01992643/document 
Armagnague-Roucher, Maitena ; Rigoni, Isabelle ; Valette, Marie-Fraçoise dir. (2018) Dossier : École et migration, Revue européenne des migrations internationales, vol. 34, nº, 248 p. https:// doi.org/10.4000/remi.11498

Armagnague-Roucher, Maitena ; Rigoni, Isabelle ; Tersigni, Simona (2019) Dossier : La scolarisation des élèves migrants en France. Migrations Société, vol. 41, n¹76. 196 p. https://doiorg.ressources.un

Audren, Gwenaelle ; Baby-Collin, Virginie ; Valcin, Marguerite (2018) L'école, une ressource pour les populations migrantes. Regards croisés de l'institution et des parents d'élèves dans le centreville de Marseille, Revue européenne des migrations internationales, vol. 4, n4, pp. 93- 118. https:// doi.org/10.4000/remi.11751

Azaoui, Brahim (2020) Enseigner auprès d'élèves en situation de grande précarité : du désir didactique à la souffrance ressentie. In Mendonça Dias, Catherine ; Azaoui, Brahim ; ChnaneDavin, Fatima. (dir), Allophonie. Inclusion et langues des enfants migrants à l'école, pp. 107-122, Lambert-Lucas.

Baby-Collin, Virginie ; Exbrayat, Cécile ; Russo, Luna (à paraitre 2021) Trajectoires scolaires et fabrique de l'orientation scolaire de lycéens migrants allophones. Le cas d'un lycée de Marseille, Jeunes et mineurs en mobilité, nº

Baby-Collin, Virginie ; Exbrayat, Cécile ; Palash, Polina ; Russo Luna (à paraitre, 2021) Covid 19, confinement et élèves migrants. Le cas de lycéens marseillais. In Baby-Collin, Virginie \& Souiah, Farida (dir). Enfances et jeunesses en migration, Le Cavalier bleu-MMSH

Bouillon, Florence ; Deboulet, Agnès ; Dietrich-Ragon, Pascale ; Fijalkow, Yankel (2019) Vulnérabilités résidentielles, Bibliothèque des territoires. De l'Aube. 263 p.

Bulletin Officiel de l'Éducation Nationale (2012) Organisation de la scolarité des EANA, Circulaire no2012-141.

Chnane-Davin, Fatima (2020) De quoi faut-il tenir compte lorsqu'on enseigne le français langue étrangère et seconde ?, In Defays, Jean-Marc, Le FLE en questions: Enseigner le français langue étrangère et seconde. pp. 149-198. https://doi.org/10.3917/mard.defay.2020.01.0149

Felouzis, Georges \& Fouquet-Chauprade, Barbara (2016) Les descendants d'immigrés à l'école, Revue française de pédagogie, vol. 2, n², pp. 5-10. https://doi.org/10.4000/rfp.4736

Guedat-Bittighoffe, Delphine (2015) La scolarisation des élèves allophones au collège : étude comparative des modalités d'organisation de quatre dispositifs d'accueil et des effets potentiels sur l'apprentissage du français, Les Sciences de l'éducation - Pour l'Ėre nouvelle, vol. 3, n³, pp. 83107. https://doi.org/10.3917/lsdle.483.0083

Mendonça Dias, Catherine \& Rigoni, Isabelle (2019) La demande d'asile : impacts sur la scolarisation de l'enfant et rôles de l'enseignant. Migrations Société, vol. 176, n², 49-63. doi: 10.3917/migra.176.0049.

Ministère de l'Éducation nationale de la Jeunesse et des Sports (2020) Note d'information 20.39, 67 909 élèves allophones nouvellement arrivés en 2018-2019: neuf sur dix bénéficient d'un soutien linguistique ou d'une scolarité dans un dispositif spécifique. Fabienne Rosenwald, Laurence Brun, DEPP-B1. Consulté le 15 octobre 2021 sur https://archives-statistiques-depp.education.gouv.fr/Default/ digital-viewer/c-47074

Ministère de l'Éducation Nationale et de la Jeunesse et des Sports, Éduscol (2021) Ressources pour l'accueil et la scolarisation des élèves allophones nouvellement arrivés (EANA). Consulté le 15 mai 2021 
sur https://eduscol.education.fr/1191/ressources-pour-l- accueil-et-la-scolarisation-des-elevesallophones-nouvellement-arrives-eana

Rigoni, Isabelle (2020) Enseigner aux allophones. Représentations du métier et pratiques de terrain. In Mendonça Dias, Catherine ; Azaoui, Brahim ; Chnane-Davin, Fatima. (dir), Allophonie. Inclusion et langues des enfants migrants à l'école, pp. 91-106, Lambert-Lucas.

Schiff, Claire (2002) Les jeunes primo-arrivants : un rapport à la société distinct de celui des minorités ethniques, VEI-enjeux, ํo131, pp. 222-231

UNESCO (2009) Principes directeurs pour l'inclusion dans l'éducation. 38 p. https:// unesdoc.unesco.org/ark:/48223/pf0000177849_fre

Vallet, Louis-André \& Caille, Jean-Paul (1996) Les élèves étrangers ou issus de l'immigration dans l'école et le collège français, Les dossiers d'éducation et formation, $n^{\circ} 67,153 \mathrm{p}$.

\section{Sitographie}

Association pour la promotion de la traduction littéraire : https://www.atlas-citl.org/ association/

Baby-Collin, Virginie ; Vinot, Julie - Espace Mendès France (2021, 25 mars) Les jeunes migrants et l'école : parcours et enjeux de scolarisation. (vidéo). YouTube. URL : https://www.youtube.com/ watch? $\mathrm{v}=1 \mathrm{Mcn} 2 \mathrm{AKnmKM} \& \mathrm{t}=2138 \mathrm{~s}$

Centre national d'enseignement à distance : https://www.cned.fr/

Collectif ACTE : https://www.collectif-acte.fr/a_propos

Migr'Avenir (2020) Les jeunes migrant·es et l'École : la parole des jeunes concernées (stream). Soundcloud. URL : https://soundcloud.com/user-112767485/les-jeunes-migrants-et-lecoleparcours-et-enjeux-de-scolarisation-la-parole-des-jeunes-concernees

\section{NOTES}

1. Accessible sur YouTube : https://www.youtube.com/watch?v=lMcn2AKnmKM\&t=2138s

2. L'inclusion définie comme « un processus visant à tenir compte de la diversité des besoins des élèves et à y répondre par une participation croissante à l'apprentissage » (UNESCO, 2009, p. 14) est apparue en 2005 dans le cadre de la scolarisation des élèves en situation de handicap. Aujourd'hui, elle englobe l'ensemble des élèves «à besoins éducatifs particuliers " (Ministère de l'Éducation nationale, de la Jeunesse et des Sports, 2021) parmi lesquels les EANA, et vise une meilleure égalité des chances.

3. Contrairement aux UPE2A-LGT, les UPE2A-LP n'ont pas vocation à l'inclusion et sont des classes fermées qui jouent un rôle de plateforme de première scolarisation en France.

4. Le lycée est devenu général et technologique depuis septembre 2021 avec l'ouverture d'une filière Sciences et technologies du management et de la gestion (STMG).

5. Si le dispositif fonctionne, en l'état, exactement tout comme une UPE2A, il n'en a pas l'appellation officielle. L'équipe du dispositif lui préfère la dénomination "dispositif d'inclusion FLS des EANA".

6. Le français langue seconde et de scolarisation désigne le français enseigné comme langue de l'institutionnel et de la scolarisation. Les élèves apprennent le français non seulement pour leur vie quotidienne mais aussi pour étudier. 
7. L'emploi du temps des EANA inscrit·es en UPE2A est évolutif. C'est-à-dire qu'au fur et à mesure de l'année les élèves peuvent intégrer davantage de cours de classe ordinaire comme physiquechimie. Tout cela est mouvant et appelle à être rediscuté chaque année en fonction de la dotation horaire du lycée, par exemple.

8. Accessible sur Souncloud: https://soundcloud.com/user-112767485/les-jeunes-migrants-etlecole-parcours-et-enjeux-de-scolarisation-la-parole-des-jeunes-concernees

9. Collectif ACTE. https://www.collectif-acte.fr/a_propos

10. Association pour la promotion de la traduction littéraire. https://www.atlas-citl.org/ association/

11. Centre national d'enseignement à distance. https://www.cned.fr/

\section{AUTEURS}

\section{LOLA BULTEL}

Ingénieure de recherche au laboratoire Migrinter dans le cadre du projet "Migration Positive" porté par l’Observatoire de la Migration des Mineurs, lola.bultel@laposte.net

\section{BONNIE COUILLET}

Etudiante en Master 2 Migrations Internationales de l'Université de Poitiers, couilletb@gmail.com

\section{LISE FAUVRE}

Etudiante en Master 2 Migrations Internationales de l'Université de Poitiers, lise.fauvre@outlook.fr

\section{LUNA RUSSO}

Doctorante, Aix-Marseille Université, laboratoires Telemme et Adef, pôle pilote AMPIRIC, luna.RUSSO@univ-amu.fr 